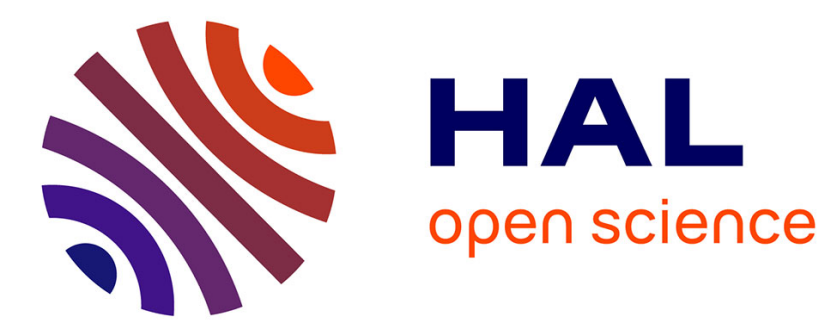

\title{
Large deviation results for wave governed random motions driven by semi-Markov processes \\ Claudio Macci
}

\section{To cite this version:}

Claudio Macci. Large deviation results for wave governed random motions driven by semi-Markov processes. Communications in Statistics - Simulation and Computation, 2011, 40 (09), pp.1342-1363. 10.1080/03610918.2011.575501 . hal-00703145

\section{HAL Id: hal-00703145 \\ https://hal.science/hal-00703145}

Submitted on 1 Jun 2012

HAL is a multi-disciplinary open access archive for the deposit and dissemination of scientific research documents, whether they are published or not. The documents may come from teaching and research institutions in France or abroad, or from public or private research centers.
L'archive ouverte pluridisciplinaire HAL, est destinée au dépôt et à la diffusion de documents scientifiques de niveau recherche, publiés ou non, émanant des établissements d'enseignement et de recherche français ou étrangers, des laboratoires publics ou privés. 


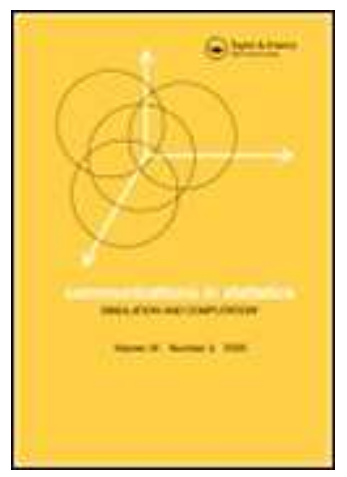

\section{Large deviation results for wave governed random motions driven \\ by semi-Markov processes}

\begin{tabular}{|r|l|}
\hline Journal: & Communications in Statistics - Simulation and Computation \\
\hline Manuscript ID: & LSSP-2010-0131.R1 \\
\hline Manuscript Type: & Original Paper \\
\hline Author: & 27-Sep-2010 \\
\hline Keywomplete List of Authors: & Macci, Claudio; Universita' di Roma, Dipartimento di Matematica \\
\hline Abstract: & $\begin{array}{l}\text { importance sampling, level crossing probability, telegrapher's } \\
\text { process }\end{array}$ \\
\hline & $\begin{array}{l}\text { In this paper we present large deviation results for a model } \\
\text { which is close to a random walk. We prove large deviation } \\
\text { estimates for level crossing probabilities. Moreover we } \\
\text { provide an asymptotically efficient simulation law for the } \\
\text { estimation of the level crossing probabilities by Monte Carlo } \\
\text { simulation based on the importance sampling technique. } \\
\text { These results will be adapted to wave governed random } \\
\text { motions driven by semi-Markov processes and we present } \\
\text { some simulations. Finally we study the convergence of } \\
\text { some large deviation rates for standard wave governed } \\
\text { random motions based on a scaling presented in the } \\
\text { literature. }\end{array}$ \\
\hline
\end{tabular}




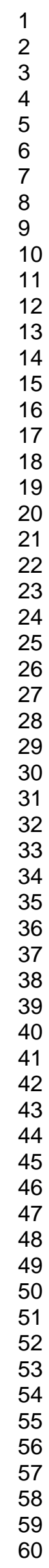

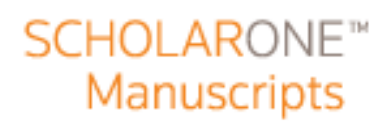

10

12

13

16

17

18

19

20

22

23

25

26

28

29

32

33

34

35

36

39

40

41

42

44

45

46

47

48

50

51 


\title{
Large deviation results for wave governed random motions driven by semi-Markov processes*
}

\author{
Claudio Macci ${ }^{\dagger}$
}

\begin{abstract}
In this paper we present large deviation results for a model $\left\{\xi_{1}+\cdots+\xi_{n}: n \geq 1\right\}$ which is close to a random walk. More precisely we consider independent random variables $\left\{\xi_{n}: n \geq 1\right\}$ such that $\left\{\xi_{n}: n \geq 2\right\}$ are i.i.d. and a different distribution for $\xi_{1}$ is allowed. We prove large deviation estimates for $P\left(N_{x} \leq x T\right)$ and $P\left(N_{x}<\infty\right)$ as $x \rightarrow \infty$, where $N_{x}:=\inf \left\{n \geq 1: \xi_{1}+\cdots+\xi_{n} \geq x\right\}$. Moreover we provide an asymptotically efficient simulation law for the estimation of $P\left(N_{x} \leq x T\right)$ and $P\left(N_{x}<\infty\right)$ by Monte Carlo simulation based on the importance sampling technique. These results will be adapted to wave governed random motions driven by semi-Markov processes and we present some simulations. Finally we study the convergence of some large deviation rates for standard wave governed random motions based on a scaling presented in the literature (see e.g. Kac (1974) and Orsingher (1990)).
\end{abstract}

Short title: Wave governed random motions driven by semi-Markov processes. Keywords: Importance sampling, level crossing probability, telegrapher's process. AMS Subject Classification: 60F10, 60K15, 65C05.

\section{Introduction}

A wave governed random motion is a two-valued integrated telegraph signal with upward velocity $c_{1}>0$ and downward velocity $-c_{2}<0$, and rates $\lambda_{1}>0$ and $\lambda_{2}>0$ of the occurrences of velocity switches, respectively (thus, in particular, we think to have two states 1 and 2 , the holding times in state 1 and in state 2 are independent, and the holding times in the state $i \in\{1,2\}$ are independent and exponentially distributed with failure rate $\lambda_{i}$ ). We remark that it is a particular continuous time Markov additive process with environment's state space $E=\{1,2\}$; see e.g. section 2.5 in Asmussen (2000) for the preliminaries on Markov additive processes (and in particular for the case with finite environment's state space).

The theory of large deviations gives an asymptotic computation of small probabilities on exponential scale; see e.g. Dembo and Zeitouni (1998) as a reference on this topic. There is a wide literature on large deviations for Markov additive processes, and the results for continuous time Markov additive processes can be applied to wave governed random motions.

In this paper we consider a more general situation, i.e. wave governed random motions driven by semi-Markov processes. There is a wide literature on semi-Markov processes with applications in several fields. Recent monographs on this topic are Janssen and Manca $(2006,2007)$ and Barbu and Limnios (2008); see also Limnios and Oprişan (2001). As we shall see in this case the holding times are independent and compound geometric distributed (and the holding times in each state are i.i.d.).

\footnotetext{
${ }^{*}$ This paper was presented in the poster session of the Actuarial and Financial Mathematics Conference (Bruxelles, February 4-5, 2010).

${ }^{\dagger}$ Dipartimento di Matematica, Università di Roma Tor Vergata, Via della Ricerca Scientifica, I-00133 Rome, Italy. e-mail: macci@mat. uniroma2.it
} 
In section 2 we present large deviation estimates for level crossing probabilities (as the level tends to infinity) and we address the problem of the estimation of the level crossing probabilities by Monte Carlo simulation. More precisely we use the importance sampling technique and we determine an asymptotically efficient simulation law. There is a wide literature on large deviations and importance sampling for the estimation by Monte Carlo simulation of rare events: here we cite Bucklew (1990, 2004), Mandjes (2007) and Asmussen and Glynn (2007). Moreover, among the papers on this topic which study the level crossing probabilities, we recall Lehtonen and Nyrhinen (1992a-b) and Stabile and Torrisi (2010).

The results in section 2 are presented for a model which is close to a random walk. In section 3 these results are applied to wave governed random motions driven by semi-Markov processes; moreover we present some simulations. In section 4 we prove the convergence of some large deviation rates for standard wave governed random motions. This kind of results appear in Macci (2009), but here we consider a different scaling in the literature (see e.g. Kac (1974) and Orsingher (1990); here we can have a non-null drift as in Beghin et al. (2001)). Finally we present an Appendix with an interesting large deviation principle.

\section{Results on large deviations and importance sampling}

In this section we present large deviation results for a model which is close to a random walk. More precisely we consider i.i.d. increments but a different distribution for the first increment is allowed. In the next section 3 we shall adapt the results in this section to wave governed random motions driven by semi-Markov processes.

In subsection 2.1 we present large deviation estimates for level crossing probabilities. In subsection 2.2 we address the problem of the estimation of the level crossing probabilities by Monte Carlo simulation. More precisely we use the importance sampling technique and we determine an asymptotically efficient simulation law.

\subsection{Large deviation results}

Let $\left\{\xi_{n}: n \geq 1\right\}$ be a sequence of independent random variables such that $\left\{\xi_{n}: n \geq 2\right\}$ are i.i.d.; moreover let $\widetilde{\kappa}$ and $\kappa$ be the functions defined by

$$
\widetilde{\kappa}(\gamma):=\log \mathbb{E}\left[e^{\gamma \xi_{1}}\right] \text { and } \kappa(\gamma):=\log \mathbb{E}\left[e^{\gamma \xi_{2}}\right] .
$$

We denote the Fenchel-Legendre transform of the function of $\kappa$ by $\kappa^{*}$, i.e. we set

$$
\kappa^{*}(x):=\sup _{\gamma \in \mathbb{R}}\{\gamma x-\kappa(\gamma)\} .
$$

The functions $\widetilde{\kappa}, \kappa$ and $\kappa^{*}$ are convex. In general, for any convex function $f$ as $\widetilde{\kappa}, \kappa$ and $\kappa^{*}$, the set

$$
\mathcal{D}(f):=\{\gamma \in \mathbb{R}: f(\gamma)<\infty\}
$$

is called domain of $f$. Moreover the interior of the set $\mathcal{D}(f)$ will be denoted by $\mathcal{D}(f)^{\circ}$.

The first result is the large deviation principle (LDP) for the sequence $\left\{\bar{\xi}_{n}: n \geq 1\right\}$, where $\bar{\xi}_{n}:=\frac{\xi_{1}+\cdots+\xi_{n}}{n}$. This LDP is a consequence of Gärtner Ellis Theorem (see e.g. Theorem 2.3.6 in Dembo and Zeitouni, 1998); in particular we refer to the concept of essentially smooth function (see e.g. Definition 2.3.5 in Dembo and Zeitouni, 1998).

Lemma 2.1 Assume that $\mathcal{D}(\kappa) \subset \mathcal{D}(\widetilde{\kappa})$ and the function $\kappa$ is essentially smooth and lower semicontinuous. Then $\left\{\bar{\xi}_{n}: n \geq 1\right\}$ satisfies the LDP with good rate function $\kappa^{*}$ defined by $(1)$; namely $\kappa^{*}$ has compact level sets,

$$
\limsup _{n \rightarrow \infty} \frac{1}{n} \log P\left(\bar{\xi}_{n} \in C\right) \leq-\inf _{x \in C} \kappa^{*}(x) \quad \text { for all closed sets } C \subset \mathbb{R}
$$


and

$$
\liminf _{n \rightarrow \infty} \frac{1}{n} \log P\left(\bar{\xi}_{n} \in G\right) \geq-\inf _{x \in G} \kappa^{*}(x) \quad \text { for all open sets } G \subset \mathbb{R} .
$$

Proof. For all $\gamma \in \mathcal{D}(\kappa)$ we have

$$
\lim _{n \rightarrow \infty} \frac{1}{n} \log \mathbb{E}\left[e^{n \gamma \bar{\xi}_{n}}\right]=\lim _{n \rightarrow \infty}\left\{\frac{\widetilde{\kappa}(\gamma)}{n}+\frac{(n-1) \kappa(\gamma)}{n}\right\}=\kappa(\gamma)
$$

and the LDP holds with an application of Gärtner Ellis Theorem.

Thus, as one expects, the different distribution of $\xi_{1}$ does not change the behavior of large deviations. However the situation could be different if $\mathcal{D}(\kappa)$ is not a subset of $\mathcal{D}(\widetilde{\kappa})$ (see the LDP presented in the Appendix).

The aim of this section is to present large deviation estimates for suitable level crossing probabilities which can be expressed in terms the random variables $\left\{N_{x}: x>0\right\}$ defined by

$$
N_{x}:=\inf \left\{n \geq 1: \xi_{1}+\cdots+\xi_{n} \geq x\right\} .
$$

In view of what follows we need to introduce some preliminaries. We consider the value

$$
w:=\sup \{\gamma \geq 0: \kappa(\gamma) \leq 0\}
$$

and the following condition

$(\mathbf{H})$ : the inclusion $\mathcal{D}(\kappa) \subset \mathcal{D}(\widetilde{\kappa})$ holds, the function $\kappa$ is strictly convex on $\mathcal{D}(\kappa)^{\circ}$ and satisfies the hypotheses of Gärtner Ellis Theorem, $\lim _{\gamma \rightarrow \infty} \kappa(\gamma)=\infty, \kappa(w)=0$ and $w \in \mathcal{D}(\kappa)^{\circ}$.

Note that $\kappa^{\prime}(w) \in[0, \infty)$, and we can have $\kappa^{\prime}(w)=0$ only if $w=0$; moreover $\kappa^{\prime}(w) \in \mathcal{D}\left(\kappa^{*}\right)^{\circ}$ by some arguments of convex analysis (see e.g. Rockafellar, 1970, as a reference on this topic).

Moreover let $R:(0, \infty) \rightarrow(0, \infty)$ be defined by

$$
R(T):= \begin{cases}T \kappa^{*}(1 / T) & \text { if } T<1 / \kappa^{\prime}(w) \\ w & \text { if } T \geq 1 / \kappa^{\prime}(w)\end{cases}
$$

where the case $T \geq 1 / \kappa^{\prime}(w)$ is empty if $\kappa^{\prime}(w)=0$; this detail will be omitted in other parts of the paper.

Furthermore, for each $\gamma \in \mathcal{D}(\kappa)$, we consider the probability measure $P_{\gamma}$ which is absolutely continuous with respect to $P$ on each $\sigma$-algebra $\mathcal{F}_{n}(n \geq 1)$, where $\mathcal{F}_{n}$ is the $\sigma$-algebra generated by $\left\{\xi_{1}, \ldots, \xi_{n}\right\}$, with density

$$
\ell_{n}^{P_{\gamma}, P}=e^{\gamma\left(\xi_{1}+\cdots+\xi_{n}\right)-\{\widetilde{\kappa}(\gamma)+(n-1) \kappa(\gamma)\}} .
$$

In what follows we use the symbol $\mathbb{E}_{P_{\gamma}}[\cdot]$ for the expected value under the law $P_{\gamma}$.

Now we are ready to prove Proposition 2.2 which provides a large deviation estimate for $P\left(N_{x} \leq\right.$ $x T)$ as $x \rightarrow \infty$; the infinite horizon case, i.e. the large deviation estimate for $P\left(N_{x}<\infty\right)$, will be presented in Remark 2.3.

Proposition 2.2 Assume that condition $(\mathbf{H})$ holds. Then we have $\lim _{x \rightarrow \infty} \frac{1}{x} \log P\left(N_{x} \leq x T\right)=$ $-R(T)$ for all $T \in(0, \infty)$ such that $1 / T \in \mathcal{D}\left(\kappa^{*}\right)^{\circ}$.

Proof. We have to prove the lower bound $\liminf _{x \rightarrow \infty} \frac{1}{x} \log P\left(N_{x} \leq x T\right) \geq-R(T)$ and the upper bound $\lim \sup _{x \rightarrow \infty} \frac{1}{x} \log P\left(N_{x} \leq x T\right) \leq-R(T)$.

Proof of the lower bound. Let us consider the set $\mathcal{E}:=\left\{s \in(0, \infty): 1 / s \in \mathcal{D}\left(\kappa^{*}\right)^{\circ}\right\}$. For all $s \in \mathcal{E} \cap(0, T]$ we have

$$
P\left(N_{x} \leq x T\right) \geq P\left(\xi_{1}+\cdots+\xi_{x s} \geq x\right)=P\left(\frac{\xi_{1}+\cdots+\xi_{x s}}{x s} \geq \frac{1}{s}\right)
$$


and, by the large deviation lower bounds in Lemma 2.1, we have

$$
\liminf _{x \rightarrow \infty} \frac{1}{x} \log P\left(N_{x} \leq x T\right) \geq \liminf _{x \rightarrow \infty} \frac{s}{x s} \log P\left(\frac{\xi_{1}+\cdots+\xi_{x s}}{x s} \geq \frac{1}{s}\right) \geq-s \inf _{z>1 / s} \kappa^{*}(z) .
$$

We have three cases: $\kappa^{\prime}(0)<0 ; \kappa^{\prime}(0) \geq 0$ and $1 / T>\kappa^{\prime}(w)$ (i.e. $1 / T>\kappa^{\prime}(0)$ since $w=0$ ); $\kappa^{\prime}(0) \geq 0$ and $1 / T \leq \kappa^{\prime}(w)$ (i.e. $1 / T \leq \kappa^{\prime}(0)$ since $w=0$ ).

- In the latter case we have $\liminf \inf _{x \rightarrow \infty} \frac{1}{x} \log P\left(N_{x} \leq x T\right) \geq 0=-R(T)$ choosing $s=T$ and noting that $\inf _{z>1 / T} \kappa^{*}(z)=\kappa^{*}\left(\kappa^{\prime}(0)\right)=0$.

- In the other cases we have $s \inf _{z>1 / s} \kappa^{*}(z)=s \kappa^{*}(1 / s)$ by the continuity of $\kappa^{*}$ at $1 / s$, and therefore we obtain

$$
\liminf _{x \rightarrow \infty} \frac{1}{x} \log P\left(N_{x} \leq x T\right) \geq-\inf _{s \in \mathcal{E} \cap(0, T]} s \kappa^{*}(1 / s) .
$$

If we consider the function $s \mapsto \gamma_{s}$ defined on $\mathcal{E}$ by $\kappa^{\prime}\left(\gamma_{s}\right)=1 / s$, we have

$$
s \kappa^{*}(1 / s)=s\left\{\frac{\gamma_{s}}{s}-\kappa\left(\gamma_{s}\right)\right\}=\gamma_{s}-s \kappa\left(\gamma_{s}\right)
$$

moreover, since $s \mapsto \gamma_{s}$ is differentiable (indeed we have $\gamma_{s}^{\prime}=\left(\kappa^{*}\right)^{\prime}(1 / s)$ by a known argument of convex analysis, see e.g. Theorem 26.5 in Rockafellar, 1970), we obtain

$$
\frac{d}{d s} s \kappa^{*}(1 / s)=\frac{d}{d s}\left\{\gamma_{s}-s \kappa\left(\gamma_{s}\right)\right\}=\gamma_{s}^{\prime}-\kappa\left(\gamma_{s}\right)-s \kappa^{\prime}\left(\gamma_{s}\right) \gamma_{s}^{\prime}=-\kappa\left(\gamma_{s}\right)
$$

We also remark that, since $\kappa^{\prime}$ is increasing by the convexity of $\kappa, \gamma_{s}$ is decreasing; moreover $\gamma_{s}=w$ for $s=1 / \kappa^{\prime}(w)$. In conclusion the derivative in (6) is negative if $s \in\left(0,1 / \kappa^{\prime}(w)\right)$ and positive if $s \in\left(1 / \kappa^{\prime}(w), \infty\right)$; thus one can check that $\inf _{s \in \mathcal{E} \cap(0, T]} s \kappa^{*}(1 / s)=R(T)$ by $(2)$ and, by (4), this completes the proof of the lower bound.

Proof of the upper bound. We start noting that

$$
P\left(N_{x} \leq x T\right)=\mathbb{E}_{P_{\gamma}}\left[\left(\ell_{N_{x}}^{P_{\gamma}, P}\right)^{-1} 1_{\left\{N_{x} \leq x T\right\}}\right]=\mathbb{E}_{P_{\gamma}}\left[e^{-\gamma\left(\xi_{1}+\cdots+\xi_{N_{x}}\right)+\left(\widetilde{\kappa}(\gamma)+\left(N_{x}-1\right) \kappa(\gamma)\right)} 1_{\left\{N_{x} \leq x T\right\}}\right] ;
$$

in what follows we prove the upper bound by setting $\gamma=w(T)$, where $w(T)$ is defined by

$$
w(T):=\left\{\begin{array}{ll}
\gamma_{T} & \text { if } T<1 / \kappa^{\prime}(w) \\
w & \text { if } T \geq 1 / \kappa^{\prime}(w),
\end{array} \quad \text { for any } T>0 \text { such that } 1 / T \in \mathcal{D}\left(\kappa^{*}\right)^{\circ} .\right.
$$

Firstly one can check that $w(T), \kappa(w(T)) \geq 0$ for any $T>0$ such that $1 / T \in \mathcal{D}\left(\kappa^{*}\right)^{\circ}$. Then, if we set $D:=e^{\widetilde{\kappa}(w(T))-\kappa(w(T))}$, we have

$$
P\left(N_{x} \leq x T\right) \leq e^{-w(T) x+\left\{\tilde{\kappa}(w(T))+\left(N_{x}-1\right) \kappa(w(T))\right\}} P_{w(T)}\left(N_{x} \leq x T\right) \leq D e^{-w(T) x+x T \kappa(w(T))},
$$

whence we obtain

$$
\begin{aligned}
\limsup _{x \rightarrow \infty} \frac{1}{x} \log P\left(N_{x} \leq x T\right) & \leq-\{w(T)-T \kappa(w(T))\} \\
& =\left\{\begin{array}{ll}
-\left(\gamma_{T}-T \kappa\left(\gamma_{T}\right)\right)=-T \kappa^{*}(1 / T) & \text { if } T<1 / \kappa^{\prime}(w) \\
-(w-T \kappa(w))=-w & \text { if } T \geq 1 / \kappa^{\prime}(w)
\end{array}=-R(T)\right.
\end{aligned}
$$

by (7), (5) with $s=T$, the equality $\kappa(w)=0$ and (2). 
Remark 2.3 Under the hypotheses of Proposition 2.2 we have $\lim _{x \rightarrow \infty} \frac{1}{x} \log P\left(N_{x}<\infty\right)=-w$; thus, in some sense, we extend the previous result to the infinite horizon case, indeed $w=R(\infty):=$ $\lim _{T \rightarrow \infty} R(T)$. This can be checked following the lines of the proof above. Firstly we note that $P\left(N_{x}<\infty\right) \geq P\left(N_{x} \leq x T\right)$ and, by the lower bound $\liminf _{x \rightarrow \infty} \frac{1}{x} \log P\left(N_{x} \leq x T\right) \geq-R(T)$ proved in Proposition 2.2, we have

$$
\liminf _{x \rightarrow \infty} \frac{1}{x} \log P\left(N_{x}<\infty\right) \geq-R(T)
$$

then we obtain the lower bound $\liminf _{x \rightarrow \infty} \frac{1}{x} \log P\left(N_{x}<\infty\right) \geq-w$ by taking taking the supremum over $T$ in (8), indeed $\sup _{T>0}-R(T)=-\inf _{T>0} R(T)=-w$. The matching upper bound $\lim \sup _{x \rightarrow \infty} \frac{1}{x} \log P\left(N_{x}<\infty\right) \leq-w$ can be proved by putting $\left\{N_{x}<\infty\right\}$ in place of $\left\{N_{x} \leq x T\right\}$ in the proof of the upper bound in Proposition 2.2; one obtains the same formulas that we had when $T \geq 1 / \kappa^{\prime}(w)$.

\subsection{Importance sampling results}

Let us start with some preliminaries on importance sampling. Let us consider $K$ independent replications of $N_{x}$ under the original law $P$; then an unbiased estimator of $\pi_{x, T}:=P\left(N_{x} \leq x T\right)$ is the relative frequency $\widehat{\pi}_{x, T}$ of the level crossings

$$
\widehat{\pi}_{x, T}:=\frac{1}{K} \sum_{i=1}^{K} 1_{\left\{N_{x}^{(i)} \leq x T\right\}}
$$

where $N_{x}^{(1)}, \ldots, N_{x}^{(K)}$ are the sampled values of $N_{x}$ in each replication.

Moreover, by Proposition 2.2, this Monte Carlo approach needs $K$ growing exponentially with $x$ to keep a fixed relative precision, indeed the relative precision of $\widehat{\pi}_{x, T}$ is

$$
\frac{1}{P\left(N_{x} \leq x T\right)} \sqrt{\frac{P\left(N_{x} \leq x T\right)\left(1-P\left(N_{x} \leq x T\right)\right)}{K}} .
$$

Thus we overcome this problem by considering $K$ independent replications under another law $P^{\circ}$ chosen in a suitable way. Firstly $P^{\circ}$ is such that $P$ is absolutely continuous with respect to $P^{\circ}$ locally on the event $\left\{N_{x} \leq x T\right\}$ with positive local density; moreover an unbiased estimator of $P\left(N_{x} \leq x T\right)$ is

$$
\left[\widehat{\pi}_{x, T}\right]_{P^{\circ}}=\frac{1}{K} \sum_{i=1}^{K} \ell_{N_{x}^{(i)}}^{P, P^{\circ}} 1_{\left\{N_{x}^{(i)} \leq x T\right\}}=\frac{1}{K} \sum_{i=1}^{K}\left(\ell_{N_{x}^{(i)}}^{P^{\circ}, P}\right)^{-1} 1_{\left\{N_{x}^{(i)} \leq x T\right\}},
$$

where in general $\ell_{N_{x}^{(i)}}^{P, P^{\circ}}$ is the local density of $P$ with respect to $P^{\circ}$. The variance of this unbiased estimator is

$$
\operatorname{Var}_{P^{\circ}}\left[\left[\widehat{\pi}_{x, T}\right]_{P^{\circ}}\right]=\frac{\operatorname{Var}_{P^{\circ}}\left[\left(\ell_{N_{x}}^{P^{\circ}, P}\right)^{-1} 1_{\left\{N_{x} \leq x T\right\}}\right]}{K}=\frac{\mathbb{E}_{P^{\circ}}\left[\left(\ell_{N_{x}}^{P^{\circ}, P}\right)^{-2} 1_{\left\{N_{x} \leq x T\right\}}\right]-\left(P\left(N_{x} \leq x T\right)\right)^{2}}{K} .
$$

The aim is to choose $P^{\circ}$ in order to minimize $\operatorname{Var}_{P^{\circ}}\left[\left[\widehat{\pi}_{x, T}\right]_{P^{\circ}}\right]$. One could think to choose $P^{\circ}$ to have a null variance but, as we see, $P^{\circ}$ should depend on the unknown quantity $P\left(N_{x} \leq x T\right)$ to estimate: we have a null variance if and only if $\left(\ell_{N_{x}}^{P^{\circ}}, P\right)^{-1}$ is constant and, by taking into account the equality $\mathbb{E}_{P^{\circ}}\left[\left(\ell_{N_{x}^{\circ}}^{P^{\circ} P}\right)^{-1} 1_{\left\{N_{x} \leq x T\right\}}\right]=P\left(N_{x} \leq x T\right)$, the constant density should be equal to $\frac{P\left(N_{x} \leq x T\right)}{P^{\circ}\left(N_{x} \leq x T\right)}$. 
In what follows we choose $P^{\circ}$ in order to minimize $\operatorname{Var}_{P^{\circ}}\left[\left[\widehat{\pi}_{x, T}\right]_{P^{\circ}}\right]$ in the sense of the criterion of Siegmund (1976). By (9) the only part of $\operatorname{Var}_{P^{\circ}}\left[\left[\widehat{\pi}_{x, T}\right]_{P^{\circ}}\right]$ which depends on $P^{\circ}$ is the second moment

$$
\eta\left(x, T ; P^{\circ}\right):=\mathbb{E}_{P^{\circ}}\left[\left(\ell_{N_{x}}^{P^{\circ}, P}\right)^{-2} 1_{\left\{N_{x} \leq x T\right\}}\right] .
$$

The minimization of this second moment for a fixed $x$ is often intractable. Thus, since in the applications we are interested in large values of $x$, we concentrate our attention on the asymptotic behavior of $\frac{1}{x} \log \eta\left(x, T ; P^{\circ}\right)$ as $x \rightarrow \infty$. In this way we can use standard features on large deviations, indeed we have

$$
\liminf _{x \rightarrow \infty} \frac{1}{x} \log \eta\left(x, T ; P^{\circ}\right) \geq \liminf _{x \rightarrow \infty} \frac{1}{x} \log \left(P\left(N_{x} \leq x T\right)\right)^{2}=-2 R(T)
$$

by Jensen's inequality and Proposition 2.2. Thus a law $P^{\circ}$ (with respect to which $P$ is absolutely continuous with respect to $P^{\circ}$ locally on the event $\left\{N_{x} \leq x T\right\}$ with positive local density) is said to be an asymptotically efficient simulation law if

$$
\lim _{x \rightarrow \infty} \frac{1}{x} \log \eta\left(x, T ; P^{\circ}\right)=-2 R(T) ;
$$

indeed, if $K$ is chosen to guarantee a fixed relative precision

$$
\frac{1}{P\left(N_{x} \leq x T\right)} \sqrt{\frac{\eta\left(x, T ; P^{\circ}\right)-\left(P\left(N_{x} \leq x T\right)\right)^{2}}{K}}
$$

for the estimator $\left[\widehat{\pi}_{x, T}\right]_{P^{\circ}}, K$ has chance of growing less than exponentially if and only if (11) holds.

Remark 2.4 (Admissible laws for the infinite horizon case) We can consider the infinite horizon case, i.e. $\pi_{x, \infty}:=P\left(N_{x}<\infty\right)$ in place of $\pi_{x, T}=P\left(N_{x} \leq x T\right)$. The presentation above can be adapted by replacing the inequality $\leq x T$ with the inequality $<\infty$; then we have an unbiased estimator $\widehat{\pi}_{x, \infty}$ and we have to refer to $\eta\left(x, \infty ; P^{\circ}\right):=\mathbb{E}_{P^{\circ}}\left[\left(\ell_{N_{x}}^{P^{\circ},{ }^{\prime}}\right)^{-2} 1_{\left\{N_{x}<\infty\right\}}\right]$. Note that we trivially have $P\left(N_{x}<\infty\right)=1$ for all $x>0$ if the net profit condition fails (i.e. if $\left.\kappa^{\prime}(0) \geq 0\right)$; so this case has no interest. On the contrary, if the net profit condition $\kappa^{\prime}(0)<0$ holds, we have a further problem: the simulation time under $P$ is not finite if the level crossing does not occur. Thus we overcome this problem choosing $P^{\circ}$ in a class of admissible laws $\mathcal{A}$ such that, for any $P^{\circ} \in \mathcal{A}$, we have $P^{\circ}\left(N_{x}<\infty\right)=1$ for all $x>0$. Therefore the simulation time under each $P^{\circ} \in \mathcal{A}$ is almost surely finite because the level crossing occurs in a finite time with probability 1.

We conclude with the results. We shall see that, for each fixed $T \in(0, \infty), P_{w(T)}$ is an asymptotically efficient simulation law for the estimation of $P\left(N_{x} \leq x T\right)$ when $x$ is large. Moreover we shall see in Remark 2.6 that the asymptotically efficient simulation law for the infinite horizon case, i.e. for the estimation of $P\left(N_{x}<\infty\right)$, is $P_{w}$ (note that $P_{w}=P_{w(\infty)}$ where $w(\infty):=\lim _{T \rightarrow \infty} w(T)$ ).

Proposition 2.5 Assume condition $(\mathbf{H})$ holds. Then we have $\lim _{x \rightarrow \infty} \frac{1}{x} \log \eta\left(x, T ; P_{w(T)}\right)=-2 R(T)$ for all $T \in(0, \infty)$ such that $1 / T \in \mathcal{D}\left(\kappa^{*}\right)^{\circ}$.

Proof. The lower bound $\liminf \inf _{x \rightarrow \infty} \frac{1}{x} \log \eta\left(x, T ; P_{w(T)}\right) \geq-2 R(T)$ holds by (10) with $P^{\circ}=$ $P_{w(T)}$. For the matching upper bound we follow the same lines of the proof of the upper bound of Proposition 2.2 ; then we have $\eta\left(x, T ; P_{w(T)}\right) \leq D^{2} e^{-2 w(T) x+2 x T \kappa(w(T))}$, whence we obtain

$$
\limsup _{x \rightarrow \infty} \frac{1}{x} \log \eta\left(x, T ; P_{w(T)}\right) \leq-2\{w(T)-T \kappa(w(T))\}=-2 R(T) .
$$

Remark 2.6 (The infinite horizon case) Let $\eta\left(x, \infty ; P_{w}\right)$ be as in Remark 2.4. Under the hypotheses of Proposition of 2.5 we have $\lim _{x \rightarrow \infty} \frac{1}{x} \log \eta\left(x, \infty ; P_{w}\right)=-2 w$. Moreover, as we said in Remark 2.4, we have to check the admissibility of $P_{w}$ if the net profit condition holds. It is known that $P_{\gamma} \in \mathcal{A}$ if and only if $\kappa^{\prime}(\gamma)>0$. Moreover the net profit condition holds if and only if $\kappa^{\prime}(0)<0$; so, by the convexity of $\kappa$, we have $w>0$ and $\kappa^{\prime}(w)>0$, and therefore $P_{w} \in \mathcal{A}$. 


\section{On wave governed random motions driven by semi-Markov pro- cesses}

In this section we illustrate how the results in section 2 can be adapted to wave governed random motions driven by semi-Markov processes. We also present some simulations.

\subsection{Preliminaries}

We start by recalling the definition of semi-Markov process. Let $J=\left\{J_{n}: n \geq 0\right\}$ be a discrete time Markov chain with finite state space $E$ with transition matrix $\left(p_{i j}: i, j \in E\right)$ and define the set $\Sigma=\left\{(i, j) \in E \times E: p_{i j}>0\right\}$. Moreover let $\left(F_{i j}:(i, j) \in \Sigma\right)$ be a family distribution functions of positive random variables. Furthermore let $S=\left\{S_{n}: n \geq 0\right\}$ be a strictly increasing sequence of nonnegative random variables such that $S_{0}=0$ and $\left\{S_{n+1}-S_{n}: n \geq 0\right\}$ are conditionally independent given $J$, with conditional distribution function

$$
P\left(S_{n+1}-S_{n} \leq t \mid J\right)=F_{J_{n} J_{n+1}}(t) \text { for all } n \geq 0 .
$$

Then $Z=\{Z(t): t \geq 0\}$ defined by

$$
Z(t)=J_{n} \text { for } t \in\left[S_{n}, S_{n+1}\right) \text { and } n \geq 0
$$

is called semi-Markov process.

Throughout this paper we consider the following particular case:

$$
\left\{\begin{array}{l}
E=\{1,2\} ; \\
\text { the distribution functions }\left(F_{i j}:(i, j) \in \Sigma\right) \text { depend on } i \text { only, and we use the symbol } F_{i} \\
\text { the transition matrix is } P=\left(\begin{array}{cc}
1-p_{1} & p_{1} \\
p_{2} & 1-p_{2}
\end{array}\right) \text { with } p_{1}, p_{2} \in(0,1] .
\end{array}\right.
$$

The cases $p_{1}=0$ and $p_{2}=0$ are avoided to have an irreducible Markov chain $J$.

Then, given $c_{1}, c_{2}>0$, a wave governed random motion $A=\{A(t): t \geq 0\}$ driven by a semi-Markov process $Z$ is a continuous and piecewise linear process such that: $A$ is linear (and increasing) with slope $c_{1}$ when $Z$ occupies the state $1 ; A$ is linear (and decreasing) with slope $-c_{2}$ when $Z$ occupies the state 2 . We remark that we recover the standard wave governed random motion if $p_{1}=p_{2}=1$ and, for each $i \in\{1,2\}, F_{i}$ is the distribution function of the exponential distribution with rate $\lambda_{i}$.

The holding times in $i \in E=\{1,2\}$ will be denoted by $\left\{Y_{n}^{(i)}: n \geq 1\right\}$. We explain how they are defined with a specific example of state selection generated by $J=\left\{J_{n}: n \geq 0\right\}$ :

$$
\left(J_{0}, J_{1}, J_{2}, J_{3}, J_{4}, J_{5}, J_{6}, J_{7}, \ldots\right)=(1,1,2,1,2,2,2,1, \ldots) .
$$

We recall that $S_{0}=0$. Then we have

$$
Z(t)=\left\{\begin{array} { l l } 
{ 1 } & { \text { if } 0 \leq t < S _ { 2 } , } \\
{ 2 } & { \text { if } S _ { 2 } \leq t < S _ { 3 } , } \\
{ 1 } & { \text { if } S _ { 3 } \leq t < S _ { 4 } , } \\
{ 2 } & { \text { if } S _ { 4 } \leq t < S _ { 7 } , } \\
{ 1 } & { \text { if } S _ { 7 } \leq t < \ldots } \\
{ \vdots } & { \vdots }
\end{array} \quad \text { and } \quad \left\{\begin{array}{l}
Y_{1}^{(1)}=\left(S_{2}-S_{1}\right)+S_{1}, \\
Y_{1}^{(2)}=S_{3}-S_{2}, \\
Y_{2}^{(1)}=S_{4}-S_{3}, \\
Y_{2}^{(2)}=S_{7}-S_{4}=\left(S_{7}-S_{6}\right)+\left(S_{6}-S_{5}\right)+\left(S_{5}-S_{4}\right), \\
Y_{3}^{(1)}=\ldots \\
\vdots
\end{array}\right.\right.
$$

where:

$Y_{1}^{(1)}$ is a sum between two independent random variables with distribution function $F_{1}$; 
$Y_{1}^{(2)}$ is a random variable with distribution function $F_{2}$;

$Y_{2}^{(1)}$ is a random variable with distribution function $F_{1}$;

$Y_{2}^{(2)}$ is a sum between three independent random variables with distribution function $F_{2}$; $Y_{3}^{(1)} \ldots$

Thus, conditionally on $J$, we have sums of i.i.d. random variables, and the distribution of each random variable depends on the occupied state; moreover the number of the random variables in each sum is given by the number of consecutive transitions of $J$ in each state. In conclusion $\left\{Y_{n}^{(i)}: n \geq 1\right\}$ are i.i.d., and they are $p_{i}$-geometric compound sums of i.i.d. random variables with distribution function $F_{i}$, i.e. they have distribution function $G_{i}=\sum_{n=1}^{\infty}\left(1-p_{i}\right)^{n-1} p_{i} F_{i}^{* n}$ (where $F_{i}^{* n}$ is the $n$-th power of $F_{i}$ in the sense of convolution) as in eq. (4.1.1) in Willmot and Lin (2001); moreover $\left\{Y_{n}^{(1)}: n \geq 1\right\}$ and $\left\{Y_{n}^{(2)}: n \geq 1\right\}$ are independent sequences.

Let $i \in E$ be arbitrarily fixed. Let $\Lambda_{i}$ be the cumulant function concerning random variables with distribution function $F_{i}$, i.e.

$$
\Lambda_{i}(\gamma):=\log \int_{0}^{\infty} e^{\gamma x} F_{i}(d x)
$$

and let $\mathcal{D}\left(\Lambda_{i}\right)$ be the domain of $\Lambda_{i}$, i.e.

$$
\mathcal{D}\left(\Lambda_{i}\right):=\left\{\gamma \in \mathbb{R}: \Lambda_{i}(\gamma)<\infty\right\}
$$

We remark that $\Lambda_{i}$ is a strictly increasing function. We also consider the (common) cumulant of the random variables $\left\{Y_{n}^{(i)}: n \geq 1\right\}$, i.e.

$$
\kappa_{i}(\gamma):=\log \mathbb{E}\left[e^{\gamma Y_{n}^{(i)}}\right]
$$

then we have $e^{\kappa_{i}(\gamma)}=\sum_{k=1}^{\infty}\left(e^{\Lambda_{i}(\gamma)}\right)^{k}\left(1-p_{i}\right)^{k-1} p_{i}$, whence we obtain (we have to distinguish the cases $p_{i} \in(0,1)$ and $p_{i}=1$, but each one leads to the same formula)

$$
\kappa_{i}(\gamma)= \begin{cases}\log \frac{p_{i} e^{\Lambda_{i}(\gamma)}}{1-e^{\Lambda_{i}(\gamma)}\left(1-p_{i}\right)} & \text { if } \gamma \in \mathcal{D}\left(\kappa_{i}\right) \\ \infty & \text { otherwise }\end{cases}
$$

where $\mathcal{D}\left(\kappa_{i}\right)$ is the domain of $\kappa_{i}$, i.e.

$$
\mathcal{D}\left(\kappa_{i}\right):=\left\{\gamma \in \mathbb{R}: \kappa_{i}(\gamma)<\infty\right\}= \begin{cases}\left\{\gamma \in \mathbb{R}: e^{\Lambda_{i}(\gamma)}\left(1-p_{i}\right)<1\right\} & \text { if } p_{i} \in(0,1) \\ \left\{\gamma \in \mathbb{R}: \Lambda_{i}(\gamma)<\infty\right\} & \text { if } p_{i}=1\end{cases}
$$

Finally note that we have $\kappa_{i}=\Lambda_{i}$ if $p_{i}=1$.

\subsection{Application of the results in section 2}

Now we specialize the results in section 2 to the wave governed random motions driven by semiMarkov processes presented above. We start by considering the sequence $\left\{\xi_{n}: n \geq 1\right\}$ defined by

$$
\xi_{1}:=c_{1} Y_{1}^{(1)} 1_{J_{0}=1}+\left(-c_{2} Y_{1}^{(2)}+c_{1} Y_{1}^{(1)}\right) 1_{J_{0}=2}=c_{1} Y_{1}^{(1)}-c_{2} Y_{1}^{(2)} 1_{J_{0}=2}
$$

and, for $n \geq 2$,

$$
\xi_{n}:=\left(-c_{2} Y_{n-1}^{(2)}+c_{1} Y_{n}^{(1)}\right) 1_{J_{0}=1}+\left(-c_{2} Y_{n}^{(2)}+c_{1} Y_{n}^{(1)}\right) 1_{J_{0}=2}=c_{1} Y_{n}^{(1)}-c_{2}\left(Y_{n-1}^{(2)} 1_{J_{0}=1}+Y_{n}^{(2)} 1_{J_{0}=2}\right) .
$$


Then the functions $\kappa$ and $\widetilde{\kappa}$ are defined by

$$
\left\{\begin{array}{l}
\kappa(\gamma):=\kappa_{1}\left(c_{1} \gamma\right)+\kappa_{2}\left(-c_{2} \gamma\right) \\
\widetilde{\kappa}(\gamma):=\log \left(e^{\kappa_{1}\left(c_{1} \gamma\right)} P\left(J_{0}=1\right)+e^{\kappa(\gamma)} P\left(J_{0}=2\right)\right)
\end{array}\right.
$$

in particular, since the domain of $\kappa$ is

$$
\mathcal{D}(\kappa):=\{\gamma \in \mathbb{R}: \kappa(\gamma)<\infty\}=\left\{\gamma \in \mathbb{R}: c_{1} \gamma \in \mathcal{D}\left(\kappa_{1}\right),-c_{2} \gamma \in \mathcal{D}\left(\kappa_{2}\right)\right\},
$$

we have

$$
\begin{aligned}
\kappa(\gamma) & = \begin{cases}\log \frac{p_{1} e^{\Lambda_{1}\left(c_{1} \gamma\right)}}{1-e^{\Lambda_{1}\left(c_{1} \gamma\right)}\left(1-p_{1}\right)}+\log \frac{p_{2} e^{\Lambda_{2}\left(-c_{2} \gamma\right)}}{1-e^{\Lambda_{2}\left(-c_{2} \gamma\right)}\left(1-p_{2}\right)} & \text { if } c_{1} \gamma \in \mathcal{D}\left(\kappa_{1}\right) \text { and }-c_{2} \gamma \in \mathcal{D}\left(\kappa_{2}\right) \\
\infty & \text { otherwise }\end{cases} \\
& = \begin{cases}\log \frac{p_{1} e^{\Lambda_{1}\left(c_{1} \gamma\right)} p_{2} e^{\Lambda_{2}\left(-c_{2} \gamma\right)}}{\left(1-e^{\Lambda_{1}\left(c_{1} \gamma\right)}\left(1-p_{1}\right)\right)\left(1-e^{\Lambda_{2}\left(-c_{2} \gamma\right)}\left(1-p_{2}\right)\right)} & \text { if } c_{1} \gamma \in \mathcal{D}\left(\kappa_{1}\right) \text { and }-c_{2} \gamma \in \mathcal{D}\left(\kappa_{2}\right) \\
\infty & \text { otherwise. }\end{cases}
\end{aligned}
$$

Note that $\mathcal{D}(\kappa)$ coincides with $\mathcal{D}(\widetilde{\kappa})$; thus $\mathcal{D}(\kappa) \subset \mathcal{D}(\widetilde{\kappa})$ trivially holds. Moreover we have $\sup \mathcal{D}(\kappa)=+\infty$ if and only if $\sup \mathcal{D}\left(\kappa_{1}\right)=+\infty$, or equivalently $p_{1}=1$ and $\sup \mathcal{D}\left(\Lambda_{1}\right)=+\infty$. Similarly we have $\inf \mathcal{D}(\kappa)=-\infty$ if and only if $\sup \mathcal{D}\left(\kappa_{2}\right)=+\infty$, or equivalently $p_{2}=1$ and $\sup \mathcal{D}\left(\Lambda_{2}\right)=+\infty$. We also have $\kappa^{\prime}(0)=c_{1} \frac{\int_{0}^{\infty} x F_{1}(d x)}{p_{1}}-c_{2} \frac{\int_{0}^{\infty} x F_{2}(d x)}{p_{2}}$ (this value appears in Remarks 2.4-2.6 when we talk about the net profit condition).

We conclude with some remarks on the laws $\left\{P_{\gamma}: \gamma \in \mathcal{D}(\kappa)\right\}$ defined by the densities $\left\{\ell_{n}^{P_{\gamma}, P}\right.$ : $n \geq 1\}$ in (3) with respect to the $\sigma$-algebras $\left\{\mathcal{F}_{n}: n \geq 1\right\}$. Firstly $\mathcal{F}_{n}$ is the $\sigma$-algebra generated by $\left\{J_{0},\left(Y_{1}^{(1)}, Y_{1}^{(2)}\right), \ldots,\left(Y_{n}^{(1)}, Y_{n}^{(2)}\right)\right\}$. Moreover $\ell_{n}^{P_{\gamma}, P}$ consists of a conditional exponential change of measures given $J_{0}$; this is quite different from what happens in other papers on large deviations for models in a Markovian environment as, for instance, Lehtonen and Nyrhinen (1992b) (see also Iscoe et al. (1985) and Ney and Nummelin (1987a-b) which concern the case with a general environment state space) where one has a unique exponential change of measure.

Finally we can provide a qualitative description of each one of the laws $\left\{P_{\gamma}: \gamma \in \mathcal{D}(\kappa)\right\}$ in terms of the random variables $\left\{Y_{n}^{(1)}: n \geq 1\right\}$ and $\left\{Y_{n}^{(2)}: n \geq 1\right\}$. This is useful for the simulations presented in the next subsection.

Lemma 3.1 For each $\gamma \in \mathcal{D}(\kappa)$, under $P_{\gamma}$ we have: $\left\{Y_{n}^{(1)}: n \geq 1\right\}$ and $\left\{Y_{n}^{(2)}: n \geq 1\right\}$ are independent; furthermore, for each fixed $i \in E,\left\{Y_{n}^{(i)}: n \geq 1\right\}$ are i.i.d. $p_{i}^{(\gamma)}$-geometric compound sums of i.i.d. random variables with distribution $Q_{i}^{(\gamma)}$, where $p_{i}^{(\gamma)}:=1-e^{\kappa_{i}\left((-1)^{i+1} c_{i} \gamma\right)}\left(1-p_{i}\right)$ and $Q_{i}^{(\gamma)}$ is absolutely continuous with respect to the original law $Q_{i}$ of $\left\{Y_{n}^{(i)}: n \geq 1\right\}$ with density $\frac{d Q_{i}^{(\gamma)}}{d Q_{i}}(x)=e^{(-1)^{i+1} c_{i} \gamma x-\kappa_{i}\left((-1)^{i+1} c_{i} \gamma\right)}$.

A generalization where $E$ has at least three points. One can wonder if it is possible to consider a generalization of the framework above where $E=\{1, \ldots, s\}$ for some $s \geq 3$. Given $c_{1}, \ldots, c_{s}>0$, a possible way is the following: $p_{i, i+1} \in(0,1]$ and $p_{i i}=1-p_{i, i+1}$ for each $i \in$ $\{1, \ldots, s-1\} ; p_{s 1} \in(0,1]$ and $p_{s s}=1-p_{s 1} ; A$ is linear (and increasing) with slope $c_{1}$ when $Z$ occupies the state $1 ; A$ is linear (and decreasing) with slope $-c_{i}$ when $Z$ occupies any state $i \in\{2, \ldots, s\}$. In such a case, if we set $q_{i}:=p_{i, i+1}$ for each $i \in\{1, \ldots, s-1\}$ and $q_{s}:=p_{s 1}$, the function $\kappa$ is defined by

$$
\kappa(\gamma):=\kappa_{1}\left(c_{1} \gamma\right)+\sum_{i=2}^{s} \kappa_{i}\left(-c_{i} \gamma\right)
$$

where, in general, $\kappa_{i}$ is the logarithm of the moment generating function of a suitable $q_{i}$-geometric compound sums of i.i.d. random variables. 


\subsection{Simulations}

In this subsection $F_{1}$ and $F_{2}$ are distribution functions of exponential random variables. So we start with some preliminaries concerning this framework. Let $i \in\{1,2\}$ be arbitrarily fixed. We set $F_{i}(x):=1-e^{-\lambda_{i} x}$ (for $x \geq 0$ ) for some $\lambda_{i}>0$; thus $F_{i}$ is the distribution function of any exponential random variable with failure rate $\lambda_{i}$. Then $\Lambda_{i}$ and $\kappa_{i}$ become

$$
\Lambda_{i}(\gamma)= \begin{cases}\log \frac{\lambda_{i}}{\lambda_{i}-\gamma} & \text { if } \gamma<\lambda_{i} \\ \infty & \text { otherwise }\end{cases}
$$

and

$$
\kappa_{i}(\gamma)=\left\{\begin{array}{ll}
\log \frac{p_{i} \frac{\lambda_{i}}{\lambda_{i}-\gamma}}{1-\frac{\lambda_{i}}{\lambda_{i}-\gamma}\left(1-p_{i}\right)} & \text { if } \frac{\lambda_{i}}{\lambda_{i}-\gamma}\left(1-p_{i}\right)<1 \\
\infty & \text { otherwise }
\end{array}= \begin{cases}\log \frac{\lambda_{i} p_{i}}{\lambda_{i} p_{i}-\gamma} & \text { if } \gamma<\lambda_{i} p_{i} \\
\infty & \text { otherwise. }\end{cases}\right.
$$

Note that, as already remarked, $\kappa_{i}=\Lambda_{i}$ if $p_{i}=1$. Finally, by (12), we have

$$
\kappa(\gamma)= \begin{cases}\log \frac{\lambda_{1} p_{1} \lambda_{2} p_{2}}{\left(\lambda_{1} p_{1}-c_{1} \gamma\right)\left(\lambda_{2} p_{2}+c_{2} \gamma\right)} & \text { if }-\frac{\lambda_{2} p_{2}}{c_{2}}<\gamma<\frac{\lambda_{1} p_{1}}{c_{1}} \\ \infty & \text { otherwise }\end{cases}
$$

We consider the following choice of the parameters:

$$
P\left(J_{0}=1\right)=1 / 3=1-P\left(J_{0}=2\right) ; p_{1}=1 / 4 ; p_{2}=1 / 2 ; \quad \lambda_{1}=2 ; \lambda_{2}=1 ; c_{1}=3 ; c_{2}=6 .
$$

The net profit condition holds since $\kappa^{\prime}(0)=\frac{c_{1}}{\lambda_{1} p_{1}}-\frac{c_{2}}{\lambda_{2} p_{2}}=-6<0$. Moreover we have $w=$ $\frac{\lambda_{1} p_{1}}{c_{1}}-\frac{\lambda_{2} p_{2}}{c_{2}}=\frac{1}{12}, \kappa^{\prime}(w)=\frac{c_{2}}{\lambda_{2} p_{2}}-\frac{c_{1}}{\lambda_{1} p_{1}}=6$ and, for $T<1 / \kappa^{\prime}(w)$, we have

$$
w(T)=\frac{1}{2}\left(\frac{\lambda_{1} p_{1}}{c_{1}}-\frac{\lambda_{2} p_{2}}{c_{2}}-2 T+\sqrt{\left(\frac{\lambda_{1} p_{1}}{c_{1}}+\frac{\lambda_{2} p_{2}}{c_{2}}\right)^{2}+4 T^{2}}\right) .
$$

It is interesting to give a brief description of the distributions $\left\{P_{\gamma}: \gamma \in \mathcal{D}(\kappa)\right\}$ in Lemma 3.1, where $\mathcal{D}(\kappa)=\left(-\frac{\lambda_{2} p_{2}}{c_{2}}, \frac{\lambda_{1} p_{1}}{c_{1}}\right)=\left(-\frac{1}{12}, \frac{1}{6}\right)$. We have $p_{1}^{(\gamma)}=1-\frac{\lambda_{1}}{\lambda_{1}-c_{1} \gamma}\left(1-p_{1}\right)=\frac{0.5-3 \gamma}{2-3 \gamma}, p_{2}^{(\gamma)}=$ $1-\frac{\lambda_{2}}{\lambda_{2}+c_{2} \gamma}\left(1-p_{2}\right)=\frac{0.5+6 \gamma}{1+6 \gamma}, Q_{1}^{(\gamma)}$ is the exponential distribution with failure rate $\lambda_{1}-c_{1} \gamma=2-3 \gamma$ and $Q_{2}^{(\gamma)}$ is the exponential distribution with failure rate $\lambda_{2}+c_{2} \gamma=1+6 \gamma$ (note that $2-3 \gamma, 1+6 \gamma>0$ for all $\gamma \in \mathcal{D}(\kappa))$.

We present different estimates of $P\left(N_{x} \leq x T\right)$ by Monte Carlo simulations with two methods: the crude Monte Carlo (CMC) under the original law, and the Importance Sampling (IS) under the asymptotically efficient simulation law $P_{w(T)}$ (according to Proposition 2.5). We also present numerical values for the variance of the estimators. We consider $x=30$ and two choices of $T$ : $T=0.1$ and $T=1$. Each estimate is based on $K=100$ simulations.

$\begin{array}{ccccc} & \text { CMC } & \text { CMC } & \text { IS } & \text { IS } \\ & \begin{array}{c}\text { probability } \\ \text { estimate }\end{array} & \begin{array}{c}\text { variance } \\ \text { estimate }\end{array} & \begin{array}{c}\text { probability } \\ \text { estimate }\end{array} & \begin{array}{c}\text { variance } \\ \text { estimate }\end{array} \\ \left(T<1 / \kappa^{\prime}(w)\right) & 0.03 & 2.91 \cdot 10^{-4} & 0.0328 & 5.2813 \cdot 10^{-5} \\ & 0.04 & 3.84 \cdot 10^{-4} & 0.0253 & 3.4258 \cdot 10^{-5} \\ & 0.02 & 1.96 \cdot 10^{-4} & 0.0323 & 4.4088 \cdot 10^{-5} \\ & \text { CMC } & \text { CMC } & \text { IS } & \text { IS } \\ T=1 & \text { probability } & \text { variance } & \text { probability } & \text { variance } \\ \left(T \geq 1 / \kappa^{\prime}(w)\right) & \text { estimate } & \text { estimate } & \text { estimate } & \text { estimate } \\ & 0.07 & 6.51 \cdot 10^{-4} & 0.0454 & 1.0562 \cdot 10^{-5} \\ & 0.03 & 2.91 \cdot 10^{-4} & 0.0514 & 1.2477 \cdot 10^{-5} \\ & 0.04 & 3.84 \cdot 10^{-4} & 0.0608 & 1.5319 \cdot 10^{-5}\end{array}$




\section{Results for the standard wave governed random motion}

In this section we consider the standard wave governed random motion, i.e. the case presented in subsection 3.3 (i.e. $F_{1}$ and $F_{2}$ are distribution functions of exponential random variables) with $p_{1}=p_{2}=1$.

In what follows Propositions 4.1 and 4.2 provide an expression for the functions $\kappa^{*}$ and $R$ defined by (1) and (2), respectively (and the function $\kappa$ is defined by (13)). As we shall see we have $\mathcal{D}\left(\kappa^{*}\right)=\mathbb{R}$ and therefore the condition $1 / T \in \mathcal{D}\left(\kappa^{*}\right)^{\circ}$ in Propositions 2.2 and 2.5 always holds.

Proposition 4.1 Let us consider $\gamma_{ \pm}(x):=\frac{1}{2}\left(\frac{\lambda_{1}}{c_{1}}-\frac{\lambda_{2}}{c_{2}}-\frac{2}{x} \pm \sqrt{\left(\frac{\lambda_{1}}{c_{1}}+\frac{\lambda_{2}}{c_{2}}\right)^{2}+\frac{4}{x^{2}}}\right)$. Then we have

$$
\kappa^{*}(x)= \begin{cases}x \gamma_{+}(x)-\log \frac{\lambda_{1} \lambda_{2}}{\left(\lambda_{1}-c_{1} \gamma_{+}(x)\right)\left(\lambda_{2}+c_{2} \gamma_{+}(x)\right)} & \text { if } x>0 \\ 2 \log \left(\lambda_{2} c_{1}+\lambda_{1} c_{2}\right)-\log \left(4 \lambda_{1} \lambda_{2} c_{1} c_{2}\right) & \text { if } x=0 \\ x \gamma_{-}(x)-\log \frac{\lambda_{1} \lambda_{2}}{\left(\lambda_{1}-c_{1} \gamma_{-}(x)\right)\left(\lambda_{2}+c_{2} \gamma_{-}(x)\right)} & \text { if } x<0\end{cases}
$$

Proof. Firstly, for each fixed $x \in \mathbb{R}$, we have $\kappa^{*}(x)=x \gamma(x)-\kappa(\gamma(x))$, where $\gamma=\gamma(x) \in\left(-\frac{\lambda_{2}}{c_{2}}, \frac{\lambda_{1}}{c_{1}}\right)$ is the unique solution of the equation $\kappa^{\prime}(\gamma)=x$ or, equivalently, $\gamma(\cdot)$ is the inverse of $\kappa^{\prime}$ (which is strictly increasing). Note that

$$
\kappa^{\prime}(\gamma)=\frac{c_{1}}{\lambda_{1}-c_{1} \gamma}-\frac{c_{2}}{\lambda_{2}+c_{2} \gamma}=\frac{2 c_{1} c_{2} \gamma+\lambda_{2} c_{1}-\lambda_{1} c_{2}}{\left(\lambda_{1}-c_{1} \gamma\right)\left(\lambda_{2}+c_{2} \gamma\right)}
$$

and we distinguish two cases.

Case $x=0$. We have the equation $\kappa^{\prime}(\gamma)=0$ which admits the solution $\gamma=\frac{\lambda_{1} c_{2}-\lambda_{2} c_{1}}{2 c_{1} c_{2}}=: \gamma(0)$; then we obtain

$$
\begin{aligned}
\kappa^{*}(0) & =0 \gamma(0)-\kappa(\gamma(0))=-\log \frac{\lambda_{1} \lambda_{2}}{\left(\lambda_{1}-c_{1} \gamma(0)\right)\left(\lambda_{2}+c_{2} \gamma(0)\right)} \\
& =\log \frac{\left(\lambda_{1}-\frac{\lambda_{1} c_{2}-\lambda_{2} c_{1}}{2 c_{2}}\right)\left(\lambda_{2}+\frac{\lambda_{1} c_{2}-\lambda_{2} c_{1}}{2 c_{1}}\right)}{\lambda_{1} \lambda_{2}}=\log \frac{\left(\frac{2 \lambda_{1} c_{2}-\lambda_{1} c_{2}+\lambda_{2} c_{1}}{2 c_{2}}\right)\left(\frac{2 \lambda_{2} c_{1}+\lambda_{1} c_{2}-\lambda_{2} c_{1}}{2 c_{1}}\right)}{\lambda_{1} \lambda_{2}} \\
& =\log \left(\frac{\left(\lambda_{2} c_{1}+\lambda_{1} c_{2}\right)^{2}}{4 \lambda_{1} \lambda_{2} c_{1} c_{2}}\right)=2 \log \left(\lambda_{2} c_{1}+\lambda_{1} c_{2}\right)-\log \left(4 \lambda_{1} \lambda_{2} c_{1} c_{2}\right) .
\end{aligned}
$$

Case $x \neq 0$. The equation $\kappa^{\prime}(\gamma)=x$ becomes

$$
2 c_{1} c_{2} \gamma+\lambda_{2} c_{1}-\lambda_{1} c_{2}=\left(\lambda_{1}-c_{1} \gamma\right)\left(\lambda_{2}+c_{2} \gamma\right) x
$$

thus we have the equation

$$
c_{1} c_{2} x \gamma^{2}-\left(\left(\lambda_{1} c_{2}-\lambda_{2} c_{1}\right) x-2 c_{1} c_{2}\right) \gamma+\lambda_{2} c_{1}-\lambda_{1} c_{2}-\lambda_{1} \lambda_{2} x=0
$$

with solutions $\gamma=\gamma_{ \pm}(x)$ as in the statement of the proposition. Now we note that we have the following inequalities:

$$
\begin{gathered}
\gamma_{+}(x)>\frac{1}{2}\left(\frac{\lambda_{1}}{c_{1}}-\frac{\lambda_{2}}{c_{2}}-\frac{2}{x}+\frac{\lambda_{1}}{c_{1}}+\frac{\lambda_{2}}{c_{2}}\right)=\frac{\lambda_{1}}{c_{1}}-\frac{1}{x}>\frac{\lambda_{1}}{c_{1}} \quad \text { if } x<0 ; \\
\gamma_{-}(x)<\frac{1}{2}\left(\frac{\lambda_{1}}{c_{1}}-\frac{\lambda_{2}}{c_{2}}-\frac{2}{x}-\left(\frac{\lambda_{1}}{c_{1}}+\frac{\lambda_{2}}{c_{2}}\right)\right)=-\frac{\lambda_{2}}{c_{2}}-\frac{1}{x}<-\frac{\lambda_{2}}{c_{2}} \quad \text { if } x>0 .
\end{gathered}
$$

Then, since $\gamma(x) \in\left(-\frac{\lambda_{2}}{c_{2}}, \frac{\lambda_{1}}{c_{1}}\right)$, we obtain the equality

$$
\gamma(x)= \begin{cases}\gamma_{+}(x) & \text { if } x>0 \\ \gamma_{-}(x) & \text { if } x<0\end{cases}
$$

and we complete the proof plugging $\gamma(x)$ into $\kappa^{*}(x)=x \gamma(x)-\kappa(\gamma(x))$. 
Proposition 4.2 Let $R$ be defined by (2) where $\kappa^{*}$ is as in Proposition 4.1. Then have $w=$ $\frac{\max \left\{\lambda_{1} c_{2}-\lambda_{2} c_{1}, 0\right\}}{c_{1} c_{2}}$ and $\frac{1}{\kappa^{\prime}(w)}=\frac{\lambda_{1} \lambda_{2}}{\left|\lambda_{1} c_{2}-\lambda_{2} c_{1}\right|}$. Thus: $w=\frac{\lambda_{1} c_{2}-\lambda_{2} c_{1}}{c_{1} c_{2}}>0$ and $\frac{1}{\kappa^{\prime}(w)}=\frac{\lambda_{1} \lambda_{2}}{\lambda_{1} c_{2}-\lambda_{2} c_{1}}$ if $\lambda_{1} c_{2}-$ $\lambda_{2} c_{1}>0 ; w=0$ and $\frac{1}{\kappa^{\prime}(w)}=\frac{\lambda_{1} \lambda_{2}}{\lambda_{2} c_{1}-\lambda_{1} c_{2}}$ if $\lambda_{1} c_{2}-\lambda_{2} c_{1} \leq 0$.

Proof. Note that the equation $\kappa(\gamma)=0$ is equivalent to $\frac{\lambda_{1} \lambda_{2}}{\left(\lambda_{1}-c_{1} \gamma\right)\left(\lambda_{2}+c_{2} \gamma\right)}=1$ and we have two solutions: $\gamma=0$ and $\gamma=\frac{\lambda_{1} c_{2}-\lambda_{2} c_{1}}{c_{1} c_{2}}$. Thus we have the two cases in the statement of the proposition for $w=\sup \{\gamma \geq 0: \kappa(\gamma) \leq 0\}$. Finally the value $1 / \kappa^{\prime}(w)$ can be computed by (14).

\subsection{On some different level crossing probabilities}

In this subsection we consider the level crossing probabilities $P\left(\tau_{x}^{A} \leq x T\right)$ and $P\left(\tau_{x}^{A}<\infty\right)$ (for $x>0$ ) where $\tau_{x}^{A}$ is defined by

$$
\tau_{x}^{A}:=\inf \{t \geq 0: A(t) \geq x\}
$$

We start recalling some known formulas and we refer to Macci (2009) where we have the same notation. Let us consider the functions $\kappa_{A}, \kappa_{A}^{*}$ and $R_{A}$ defined as follows:

$$
\kappa_{A}(\gamma):=\frac{-\left[\left(\lambda_{1}+\lambda_{2}\right)+\gamma\left(c_{2}-c_{1}\right)\right]+\sqrt{\Delta(\gamma)}}{2}
$$

where

$$
\begin{gathered}
\Delta(\gamma):=\left(\lambda_{1}+\lambda_{2}\right)^{2}+\gamma^{2}\left(c_{1}+c_{2}\right)^{2}+2 \gamma\left(c_{1}+c_{2}\right)\left(\lambda_{2}-\lambda_{1}\right) ; \\
\kappa_{A}^{*}(x):=\sup _{\gamma \in \mathbb{R}}\left\{\gamma x-\kappa_{A}(\gamma)\right\}, \quad \text { i.e. } \kappa_{A}^{*}(x)= \begin{cases}\left(\sqrt{\lambda_{1} \frac{x+c_{2}}{c_{1}+c_{2}}}-\sqrt{\lambda_{2} \frac{c_{1}-x}{c_{1}+c_{2}}}\right)^{2} & \text { if } x \in\left[-c_{2}, c_{1}\right] \\
\infty & \text { otherwise; }\end{cases} \\
R_{A}(T):= \begin{cases}\infty & \text { if } 0<T<\frac{1}{c_{1}} \\
\kappa_{A}^{*}\left(c_{1}\right) / c_{1}=\lambda_{1} / c_{1} & \text { if } T=\frac{1}{c_{1}} \\
T \kappa_{A}^{*}(1 / T) & \text { if } \frac{1}{c_{1}}<\frac{1}{\kappa_{A}^{\prime}\left(w_{A}\right)} \\
w_{A} & \text { if } T \geq \frac{1}{\kappa^{\prime}\left(w_{A}\right)},\end{cases}
\end{gathered}
$$

where: $w_{A}=\frac{\lambda_{1} c_{2}-\lambda_{2} c_{1}}{c_{1} c_{2}}>0$ and $\frac{1}{\kappa_{A}^{\prime}\left(w_{A}\right)}=\frac{\lambda_{1} c_{2}^{2}+\lambda_{2} c_{1}^{2}}{c_{1} c_{2}\left(\lambda_{1} c_{2}-\lambda_{2} c_{1}\right)}$ if the net profit condition $\lambda_{2} c_{1}-\lambda_{1} c_{2}<0$ holds; $w_{A}=0$ and $\frac{1}{\kappa_{A}^{\prime}\left(w_{A}\right)}=\frac{\lambda_{1}+\lambda_{2}}{\lambda_{2} c_{1}-\lambda_{1} c_{2}}$ if the net profit condition fails (i.e. if $\lambda_{2} c_{1}-\lambda_{1} c_{2} \geq 0$ ).

We also recall that

$$
\lim _{x \rightarrow \infty} \frac{1}{x} \log P\left(\tau_{x}^{A} \leq x T\right)= \begin{cases}-\infty & \text { if } T=1 / c_{1} \text { and } P\left(J_{0}=1\right)=0 \\ -R_{A}(T) & \text { otherwise }\end{cases}
$$

by Proposition 3.1 in Macci (2009) (note that $R_{A}\left(1 / c_{1}\right)=\lambda_{1} / c_{1}$ ) and, following the lines of Remark 2.3 to obtain the analogous infinite horizon results, we have $\lim _{x \rightarrow \infty} \frac{1}{x} \log P\left(\tau_{x}^{A}<\infty\right)=-w_{A}$; this limit agrees with Theorem 4.1 in Baldi and Piccioni (1999), which provides a more general result under the net profit condition, or equivalently under the condition $w_{A}>0$.

We conclude noting that $R_{A}(T)$ above and $R(T)$ in Proposition 4.2 do not coincide; this is not surprising since $P\left(\tau_{x}^{A} \leq x T\right)$ and $P\left(N_{x} \leq x T\right)$ do not coincide as well. On the contrary we have $w_{A}=w$ which meets the equality $P\left(\tau_{x}^{A}<\infty\right)=P\left(N_{x}<\infty\right)$.

\subsection{A convergence for the large deviation rates $\kappa_{A}^{*}(\cdot)$ and $R_{A}(\cdot)$}

Convergence results of large deviation rates based on a scaling in the paper of Mazza and Rullière (2004) are presented in Macci (2009). Here we consider the same kind of convergence of large deviation rates based on a different scaling presented in Orsingher (1990, section 4); see also Kac (1974) cited therein. In these papers they consider $c_{1}=c_{2}=c$ (say) and $\lambda_{1}=\lambda_{2}=\lambda$ (say), with 
$\lambda \rightarrow \infty$ and $c^{2} / \lambda \rightarrow \sigma^{2}$; thus, if we consider a moving particle with position $A(t)$ at time $t$, the first limit means that the velocity changes occur continuously (i.e. they let go to infinity the velocity changes), and the second limit implies that also the speed of the moving particle goes to infinity. Therefore the limit behavior becomes similar to the one of a Brownian motion with null drift and variance parameter $\sigma^{2}$.

We remark that the convergence of large deviation rates in this paper concerns a general choice of $c_{1}, c_{2}, \lambda_{1}, \lambda_{2}>0$ and we can have a non-null drift as in Beghin et al. (2001). More precisely for the drift and the variance parameter we have to consider $\kappa_{A}^{\prime}(0)=\frac{\lambda_{2} c_{1}-\lambda_{1} c_{2}}{\lambda_{1}+\lambda_{2}}$ and $\kappa_{A}^{\prime \prime}(0)=\frac{2 \lambda_{1} \lambda_{2}\left(c_{1}+c_{2}\right)^{2}}{\left(\lambda_{1}+\lambda_{2}\right)^{3}}$; they meet the above quantities in the papers of Kac (1974) and Orsingher (1990) if $c_{1}=c_{2}=c$ (say) and $\lambda_{1}=\lambda_{2}=\lambda$ (say), indeed we have $\kappa_{A}^{\prime}(0)=0$ and $\kappa_{A}^{\prime \prime}(0)=c^{2} / \lambda$. We remark that $\kappa_{A}^{\prime}(0)$ and $\kappa_{A}^{\prime \prime}(0)$ can be expressed in terms of $\beta, c^{\prime}, \lambda^{\prime}$ in equations (3.6), (3.8) and (3.9) in Beghin et al. (2001); more precisely we have $\kappa_{A}^{\prime}(0)=-\beta$ and $\kappa_{A}^{\prime \prime}(0)=\frac{\left(c^{\prime}\right)^{2}}{\lambda^{\prime}}$.

The results presented below are obtained by taking the limit under the following condition for some $\mu \in \mathbb{R}$ and $\sigma>0$ :

$$
(*):\left\{\begin{array}{l}
\lambda_{1}, \lambda_{2} \rightarrow \infty \\
c_{1}=c_{1}\left(\lambda_{1}, \lambda_{2}\right):=+\mu+\frac{\sigma}{\sqrt{2}} \sqrt{\lambda_{1}+\lambda_{2}} \sqrt{\frac{\lambda_{1}}{\lambda_{2}}} \\
c_{2}=c_{2}\left(\lambda_{1}, \lambda_{2}\right):=-\mu+\frac{\sigma}{\sqrt{2}} \sqrt{\lambda_{1}+\lambda_{2}} \sqrt{\frac{\lambda_{2}}{\lambda_{1}}}
\end{array}\right.
$$

Note that $c_{1}\left(\lambda_{1}, \lambda_{2}\right), c_{2}\left(\lambda_{1}, \lambda_{2}\right) \rightarrow \infty$ as $\lambda_{1}, \lambda_{2} \rightarrow \infty$ and, moreover, we have the following equalities for the first two derivatives of $\kappa$ at the origin with $c_{1}=c_{1}\left(\lambda_{1}, \lambda_{2}\right)$ and $c_{2}=c_{2}\left(\lambda_{1}, \lambda_{2}\right)$ :

$$
\kappa_{A}^{\prime}(0)=\frac{\lambda_{2} c_{1}\left(\lambda_{1}, \lambda_{2}\right)-\lambda_{1} c_{2}\left(\lambda_{1}, \lambda_{2}\right)}{\lambda_{1}+\lambda_{2}}=\mu \quad \text { and } \quad \kappa_{A}^{\prime \prime}(0)=\frac{2 \lambda_{1} \lambda_{2}\left(c_{1}\left(\lambda_{1}, \lambda_{2}\right)+c_{2}\left(\lambda_{1}, \lambda_{2}\right)\right)^{2}}{\left(\lambda_{1}+\lambda_{2}\right)^{3}}=\sigma^{2} .
$$

The corresponding items concerning the Brownian motion are well known (for instance one can adapt Proposition 2.1 in Macci (2009) to Brownian motion). Let us consider $\widetilde{\kappa}_{A}(\gamma):=\mu \gamma+\frac{\sigma^{2}}{2} \gamma^{2}$ and

$$
\widetilde{\kappa}_{A}^{*}(x):=\sup _{\gamma \in \mathbb{R}}\left\{\gamma x-\widetilde{\kappa}_{A}(\gamma)\right\}, \quad \text { i.e. } \widetilde{\kappa}_{A}^{*}(x):=\frac{(x-\mu)^{2}}{2 \sigma^{2}} .
$$

Moreover the level crossing probability decay rate is

$$
\widetilde{R}_{A}(T)= \begin{cases}T \widetilde{\kappa}_{A}^{*}(1 / T) & \text { if } 0<T<\frac{1}{\widetilde{w}_{A}^{\prime}\left(\widetilde{w}_{A}\right)} \\ \widetilde{w}_{A} & \text { if } T \geq \widetilde{\kappa}_{A}^{\prime}\left(\widetilde{w}_{A}\right)\end{cases}
$$

where: $\widetilde{w}_{A}=-\frac{2 \mu}{\sigma^{2}}>0$ and $\frac{1}{\widetilde{\kappa}_{A}^{\prime}\left(\widetilde{w}_{A}\right)}=-\frac{1}{\mu}$ if the net profit condition $\mu<0$ holds; $\widetilde{w}_{A}=0$ and $\frac{1}{\widetilde{\kappa}_{A}^{\prime}\left(\widetilde{w}_{A}\right)}=\frac{1}{\mu}$ if the net profit condition fails (i.e. if $\mu \geq 0$ ).

Proposition 4.3 Under condition $(*)$ we have $\lim \kappa_{A}^{*}(x)=\widetilde{\kappa}_{A}^{*}(x)$ for all $x \in \mathbb{R}$.

Proof. Let us consider the values of $c_{1}$ and $c_{2}$ in $(*)$. Then, at least eventually, i.e. for $\lambda_{1}, \lambda_{2}$ large enough, for all $x$ we have

$$
\begin{aligned}
\kappa_{A}^{*}(x) & =\left(\sqrt{\lambda_{1} \frac{x+c_{2}}{c_{1}+c_{2}}}-\sqrt{\lambda_{2} \frac{c_{1}-x}{c_{1}+c_{2}}}\right)^{2}=\frac{\left(\sqrt{\lambda_{1}\left(x+c_{2}\right)}-\sqrt{\lambda_{2}\left(c_{1}-x\right)}\right)^{2}}{c_{1}+c_{2}} \\
& =\frac{\left(\lambda_{1}\left(x+c_{2}\right)-\lambda_{2}\left(c_{1}-x\right)\right)^{2}}{\left(c_{1}+c_{2}\right)\left(\sqrt{\lambda_{1}\left(x+c_{2}\right)}+\sqrt{\lambda_{2}\left(c_{1}-x\right)}\right)^{2}}=\frac{\left(\left(\lambda_{1}+\lambda_{2}\right) x-\left(\lambda_{2} c_{1}-\lambda_{1} c_{2}\right)\right)^{2}}{\left(c_{1}+c_{2}\right)\left(\sqrt{\lambda_{1}\left(x+c_{2}\right)}+\sqrt{\lambda_{2}\left(c_{1}-x\right)}\right)^{2}} \\
& =\frac{\left(\lambda_{1}+\lambda_{2}\right)^{2}(x-\mu)^{2}}{\left(c_{1}+c_{2}\right)\left(\sqrt{\lambda_{1}\left(x+c_{2}\right)}+\sqrt{\lambda_{2}\left(c_{1}-x\right)}\right)^{2}}=\widetilde{\kappa}_{A}^{*}(x) \frac{2 \sigma^{2}\left(\lambda_{1}+\lambda_{2}\right)^{2}}{\left(c_{1}+c_{2}\right)\left(\sqrt{\lambda_{1}\left(x+c_{2}\right)}+\sqrt{\lambda_{2}\left(c_{1}-x\right)}\right)^{2}} .
\end{aligned}
$$


Thus, for all $x \in \mathbb{R}$, one has to check

$$
\frac{2 \sigma^{2}\left(\lambda_{1}+\lambda_{2}\right)^{2}}{\left(c_{1}+c_{2}\right)\left(\sqrt{\lambda_{1}\left(x+c_{2}\right)}+\sqrt{\lambda_{2}\left(c_{1}-x\right)}\right)^{2}}=\frac{4 \lambda_{1} \lambda_{2}\left(c_{1}+c_{2}\right)}{\left(\lambda_{1}+\lambda_{2}\right)\left(\sqrt{\lambda_{1}\left(x+c_{2}\right)}+\sqrt{\lambda_{2}\left(c_{1}-x\right)}\right)^{2}} \rightarrow 1,
$$

where the equality in (15) is a consequence of $\sigma^{2}=\frac{2 \lambda_{1} \lambda_{2}\left(c_{1}+c_{2}\right)^{2}}{\left(\lambda_{1}+\lambda_{2}\right)^{3}}$. The limit (15) holds noting that $c_{1}+c_{2}=\frac{\sigma}{\sqrt{2}} \sqrt{\lambda_{1}+\lambda_{2}} \frac{\lambda_{1}+\lambda_{2}}{\sqrt{\lambda_{1} \lambda_{2}}}$ and, for all $x \in \mathbb{R}, \sqrt{\lambda_{1}\left(x+c_{2}\right)}+\sqrt{\lambda_{2}\left(c_{1}-x\right)}$ behaves like $2 \sqrt{\frac{\sigma}{\sqrt{2}} \sqrt{\lambda_{1}+\lambda_{2}} \sqrt{\lambda_{1} \lambda_{2}}}$.

Proposition 4.4 Under condition $(*)$ we have $\lim R_{A}(T)=\widetilde{R}_{A}(T)$ for all $T \in(0, \infty)$.

Proof. Firstly note that, putting $c_{1}=c_{1}\left(\lambda_{1}, \lambda_{2}\right)$ and $c_{2}=c_{2}\left(\lambda_{1}, \lambda_{2}\right)$, we have $\lambda_{2} c_{1}-\lambda_{1} c_{2}=$ $\left(\lambda_{1}+\lambda_{2}\right) \mu$. The limit $T \kappa_{A}^{*}(1 / T) \rightarrow T \widetilde{\kappa}_{A}^{*}(1 / T)$ is a consequence of Proposition 4.3. Thus we have to check the limits

$$
w_{A} \rightarrow \widetilde{w}_{A} \text { and } \frac{1}{\kappa_{A}^{\prime}\left(w_{A}\right)} \rightarrow \frac{1}{\widetilde{\kappa}_{A}^{\prime}\left(\widetilde{w}_{A}\right)}
$$

The limits hold trivially as equalities if $\lambda_{2} c_{1}-\lambda_{1} c_{2}=\left(\lambda_{1}+\lambda_{2}\right) \mu \geq 0$, or equivalently $\mu \geq 0$, indeed we have

$$
w_{A}=0=\widetilde{w}_{A} \text { and } \frac{1}{\kappa_{A}^{\prime}\left(w_{A}\right)}=\frac{\lambda_{1}+\lambda_{2}}{\lambda_{2} c_{1}-\lambda_{1} c_{2}}=\frac{1}{\mu}=\frac{1}{\widetilde{\kappa}_{A}^{\prime}\left(\widetilde{w}_{A}\right)} .
$$

The limits also hold if $\lambda_{2} c_{1}-\lambda_{1} c_{2}=\left(\lambda_{1}+\lambda_{2}\right) \mu<0$, or equivalently $\mu<0$, indeed we have

$$
w_{A}=\frac{\lambda_{1} c_{2}-\lambda_{2} c_{1}}{c_{1} c_{2}}=\frac{-\left(\lambda_{1}+\lambda_{2}\right) \mu}{-\mu^{2}+\mu \frac{\sigma}{\sqrt{2}} \sqrt{\lambda_{1}+\lambda_{2}} \frac{\lambda_{2}-\lambda_{1}}{\sqrt{\lambda_{1} \lambda_{2}}}+\frac{\sigma^{2}}{2}\left(\lambda_{1}+\lambda_{2}\right)} \rightarrow-\frac{2 \mu}{\sigma^{2}}=\widetilde{w}_{A}
$$

and

$$
\begin{aligned}
\frac{1}{\kappa_{A}^{\prime}\left(w_{A}\right)} & =\frac{\lambda_{1} c_{2}^{2}+\lambda_{2} c_{1}^{2}}{c_{1} c_{2}\left(\lambda_{1} c_{2}-\lambda_{2} c_{1}\right)} \\
& =\frac{\left(\lambda_{1}+\lambda_{2}\right)\left(\mu^{2}+\frac{\sigma^{2}}{2}\left(\lambda_{1}+\lambda_{2}\right)\right)}{\left(-\mu^{2}+\mu \frac{\sigma}{\sqrt{2}} \sqrt{\lambda_{1}+\lambda_{2}} \frac{\lambda_{2}-\lambda_{1}}{\sqrt{\lambda_{1} \lambda_{2}}}+\frac{\sigma^{2}}{2}\left(\lambda_{1}+\lambda_{2}\right)\right)\left(-\left(\lambda_{1}+\lambda_{2}\right) \mu\right)} \rightarrow-\frac{1}{\mu}=\frac{1}{\widetilde{\kappa}_{A}^{\prime}\left(\widetilde{w}_{A}\right)} .
\end{aligned}
$$

\section{Appendix: A case where $\mathcal{D}(\kappa)$ is not a subset of $\mathcal{D}(\widetilde{\kappa})$}

Assume that $\xi_{1}$ is exponentially distributed with mean $1 / \lambda_{1}$ and $\left\{\xi_{n}: n \geq 2\right\}$ are exponentially distributed with mean $1 / \lambda_{2}$. Then we have

$$
\widetilde{\kappa}(\gamma)=\left\{\begin{array}{ll}
\log \frac{\lambda_{1}}{\lambda_{1}-\gamma} & \text { if } \gamma<\lambda_{1} \\
\infty & \text { if } \gamma \geq \lambda_{1}
\end{array} \text { and } \kappa(\gamma)= \begin{cases}\log \frac{\lambda_{2}}{\lambda_{2}-\gamma} & \text { if } \gamma<\lambda_{2} \\
\infty & \text { if } \gamma \geq \lambda_{2}\end{cases}\right.
$$

thus the domains are $\mathcal{D}(\widetilde{\kappa})=\left(-\infty, \lambda_{1}\right)$ and $\mathcal{D}(\kappa)=\left(-\infty, \lambda_{2}\right)$.

Here we assume that $\lambda_{2}>\lambda_{1}$ and therefore the inclusion $\mathcal{D}(\kappa) \subset \mathcal{D}(\widetilde{\kappa})$ fails. In this case we have

$$
\Lambda(\gamma):=\lim _{n \rightarrow \infty} \frac{1}{n} \log \mathbb{E}\left[e^{n \gamma \bar{\xi}_{n}}\right]= \begin{cases}\log \frac{\lambda_{2}}{\lambda_{2}-\gamma} & \text { if } \gamma<\lambda_{1} \\ \infty & \text { if } \gamma \geq \lambda_{1}\end{cases}
$$

(thus $\Lambda$ coincides with $\kappa$ on $\mathcal{D}(\Lambda)=\mathcal{D}(\widetilde{\kappa})=\left(-\infty, \lambda_{1}\right)$ which is a subset of $\mathcal{D}(\kappa)=\left(-\infty, \lambda_{2}\right)$ ). Note that $\Lambda$ is not essentially smooth because $\lim _{\gamma \uparrow \lambda_{1}} \Lambda^{\prime}(\gamma)=1 /\left(\lambda_{2}-\lambda_{1}\right) \in(0, \infty)$ and therefore $\Lambda$ is not steep. 
Now let us see in details the bounds provided by Gärtner Ellis Theorem. Then, if we consider the function $\Lambda^{*}$ defined by $\Lambda^{*}(x):=\sup _{\gamma \in \mathbb{R}}\{\gamma x-\Lambda(\gamma)\}$ and the set of exposed points of $\Lambda^{*}$ denoted by $F$ (see e.g. Definition 2.3.3 in Dembo and Zeitouni, 1998), we have have the following bounds:

$$
\begin{aligned}
& \limsup _{n \rightarrow \infty} \frac{1}{n} \log P\left(\bar{\xi}_{n} \in C\right) \leq-\inf _{x \in C} \Lambda^{*}(x) \quad \text { for all closed sets } C \subset \mathbb{R} ; \\
& \liminf _{n \rightarrow \infty} \frac{1}{n} \log P\left(\bar{\xi}_{n} \in G\right) \geq-\inf _{x \in G \cap F} \Lambda^{*}(x) \text { for all open sets } G \subset \mathbb{R} .
\end{aligned}
$$

We recall that, roughly speaking, $x$ is an exposed point of $\Lambda^{*}$ if $\Lambda^{*}(x)<\infty$ and $\Lambda^{*}$ is strictly convex at $x$. Then, since we have

$$
\Lambda^{*}(x)= \begin{cases}\infty & \text { if } x<0 \\ \lambda_{2} x-1-\log \left(\lambda_{2} x\right) & \text { if } 0<x<1 /\left(\lambda_{2}-\lambda_{1}\right) \\ \lambda_{1} x-\log \frac{\lambda_{2}}{\lambda_{2}-\lambda_{1}} & \text { if } x \geq 1 /\left(\lambda_{2}-\lambda_{1}\right),\end{cases}
$$

the set of exposed points is $F=\left(0,1 /\left(\lambda_{2}-\lambda_{1}\right)\right)$.

Thus we have to prove the lower bound for open sets. By the condition with eq. (1.2.8) in Dembo and Zeitouni (1998) it is enough to check that, for each fixed $x, \varepsilon>0$, we have

$$
\liminf _{n \rightarrow \infty} \frac{1}{n} \log P\left(\bar{\xi}_{n} \in(x-\varepsilon, x+\varepsilon)\right) \geq-\Lambda^{*}(x) .
$$

We distinguish two cases: $x \in F$ and $x \in(0, \infty) \backslash F$. In the first case, for $\eta \in(0, \varepsilon)$ small enough such that $(x-\eta, x+\eta) \subset F$, we have

$$
\begin{aligned}
\liminf _{n \rightarrow \infty} \frac{1}{n} \log P\left(\bar{\xi}_{n} \in(x-\varepsilon, x+\varepsilon)\right) & \geq \liminf _{n \rightarrow \infty} \frac{1}{n} \log P\left(\bar{\xi}_{n} \in(x-\eta, x+\eta)\right) \\
& \geq-\inf _{y \in(x-\eta, x+\eta) \cap F} \Lambda^{*}(y)=-\inf _{y \in(x-\eta, x+\eta)} \Lambda^{*}(y) ;
\end{aligned}
$$

therefore we obtain (16) letting $\eta$ go to zero because the function $\Lambda^{*}$ is continuous on $(0, \infty)$. Thus, from now on, we consider the second case $x \in\left[1 /\left(\lambda_{2}-\lambda_{1}\right), \infty\right)$. We start noting that, for $\eta \in(0, \varepsilon)$ and $\alpha \in(0,1)$, we have

$$
\begin{aligned}
P\left(\bar{\xi}_{n} \in(x-\eta, x+\eta)\right) & =P\left(n(x-\eta)<\sum_{i=1}^{n} \xi_{i}<n(x+\eta)\right) \\
& \geq P\left(\alpha n(x-\eta)<\xi_{1}<\alpha n(x+\eta)\right) P\left(\frac{(1-\alpha) n(x-\eta)}{n-1}<\frac{\sum_{i=2}^{n} \xi_{i}}{n-1}<\frac{(1-\alpha) n(x+\eta)}{n-1}\right) \\
& =e^{-\lambda_{1} \alpha n(x+\eta)}\left(e^{\lambda_{1} \alpha n 2 \eta}-1\right) P\left(\frac{(1-\alpha) n(x-\eta)}{n-1}<\frac{\sum_{i=2}^{n} \xi_{i}}{n-1}<\frac{(1-\alpha) n(x+\eta)}{n-1}\right) .
\end{aligned}
$$

Now, for any $\delta>0$ small enough, we can find $\bar{n}_{\delta}$ such that for all $n \geq \bar{n}_{\delta}$ we have

$P\left(\frac{(1-\alpha) n(x-\eta)}{n-1}<\frac{\sum_{i=2}^{n} \xi_{i}}{n-1}<\frac{(1-\alpha) n(x+\eta)}{n-1}\right) \geq P\left((1-\alpha)(x-\eta)-\delta<\frac{\sum_{i=2}^{n} \xi_{i}}{n-1}<(1-\alpha)(x+\eta)\right)$

moreover, by Cramér Theorem (see e.g. Theorem 2.2.3 in Dembo and Zeitouni, 1998), we have

$\liminf _{n \rightarrow \infty} \frac{1}{n} \log P\left((1-\alpha)(x-\eta)-\delta<\frac{\sum_{i=2}^{n} \xi_{i}}{n-1}<(1-\alpha)(x+\eta)\right) \geq-\inf _{y \in((1-\alpha)(x-\eta)-\delta,(1-\alpha)(x+\eta))} \kappa^{*}(y)$

where $\kappa^{*}$ is defined by (1), and therefore we have $\kappa^{*}(y)=\lambda_{2} y-1-\log \left(\lambda_{2} y\right)$ for $y>0$. Now we remark that

$$
\inf _{y \in((1-\alpha)(x-\eta)-\delta,(1-\alpha)(x+\eta))} \kappa^{*}(y) \leq \kappa^{*}((1-\alpha)(x+\eta))
$$


since $x \geq 1 /\left(\lambda_{2}-\lambda_{1}\right)$. Thus we get the inequality

$$
\liminf _{n \rightarrow \infty} \frac{1}{n} \log P\left(\frac{(1-\alpha) n(x-\eta)}{n-1}<\frac{\sum_{i=2}^{n} \xi_{i}}{n-1}<\frac{(1-\alpha) n(x+\eta)}{n-1}\right) \geq-\kappa^{*}((1-\alpha)(x+\eta))
$$

whence we obtain

$$
\begin{aligned}
\liminf _{n \rightarrow \infty} \frac{1}{n} \log P\left(\bar{\xi}_{n} \in(x-\eta, x+\eta)\right) & \geq-\lambda_{1} \alpha(x+\eta)+2 \lambda_{1} \alpha \eta-\kappa^{*}((1-\alpha)(x+\eta)) \\
& \geq-\lambda_{1} \alpha(x+\eta)+2 \lambda_{1} \alpha \eta-\{(1-\alpha)(x+\eta)-1-\log ((1-\alpha)(x+\eta))\} .
\end{aligned}
$$

Moreover, by taking the minimum of the latter right hand side with respect to $\alpha$, we have

$$
\liminf _{n \rightarrow \infty} \frac{1}{n} \log P\left(\bar{\xi}_{n} \in(x-\eta, x+\eta)\right) \geq-\lambda_{1}(x-\eta)+\log \left(\frac{\lambda_{2}(x+\eta)}{\lambda_{2}(x+\eta)-\lambda_{1}(x-\eta)}\right)
$$

(actually the minimum is achieved by taking $\left.\alpha=1-\frac{1}{\lambda_{2}(x+\eta)-\lambda_{1}(x-\eta)}\right)$, and therefore

$$
\begin{aligned}
\liminf _{n \rightarrow \infty} \frac{1}{n} \log P\left(\bar{\xi}_{n} \in(x-\varepsilon, x+\varepsilon)\right) & \geq \liminf _{n \rightarrow \infty} \frac{1}{n} \log P\left(\bar{\xi}_{n} \in(x-\eta, x+\eta)\right) \\
& \geq-\lambda_{1}(x-\eta)+\log \left(\frac{\lambda_{2}(x+\eta)}{\lambda_{2}(x+\eta)-\lambda_{1}(x-\eta)}\right) .
\end{aligned}
$$

In conclusion we obtain (16) letting $\eta$ go to zero because we have $\Lambda^{*}(x)=\lambda_{1} x-\log \frac{\lambda_{2}}{\lambda_{2}-\lambda_{1}}$ for $x \geq 1 /\left(\lambda_{2}-\lambda_{1}\right)$.

Acknowledgements. I thank Gabriele Stabile for simulations and Enzo Orsingher for giving me a copy of his paper with L. Beghin and L. Nieddu. I also thank the reviewers of the paper for their useful comments which led to an improvement of the presentation: a referee suggested the presentation of the results by referring to the model close to the random walk. The example in the Appendix appears in the notes for the Summer School SMI (Cortona, 1997) taught by Paolo Baldi. The financial support of the Research Grant PRIN 2008 Probability and Finance is gratefully acknowledged.

\section{References}

[1] Asmussen, S. (2000) Ruin Probabilities. World Scientific, Sigapore.

[2] Asmussen, S., Glynn P.W. (2007) Stochastic Simulation: Algorithms and Analysis. Springer, New York.

[3] Baldi, P., Piccioni, M. (1999) Importance sampling for continuous time Markov chains and applications to fluid models. Methodol. Comput. Appl. Probab. 1(4):375-390.

[4] Barbu, V., Limnios, N. (2008) Semi-Markov Chains and Hidden Semi-Markov Models toward Applications. Their Use in Reliability and DNA Analysis. Springer, New York.

[5] Beghin, L., Nieddu, L., Orsingher, E. (2001) Probabilistic analysis of the telegrapher's process with drift by means of relativistic transformations. Special Issue: Advances in Applied Stochastics. J.H. Dshalalow Editor. J. Appl. Math. Stochastic Anal. 14(1):11-25.

[6] Bucklew, J.A. (1990) Large Deviation Techniques in Decision, Simulation, and Estimation. John Wiley and Sons, New York. 
[7] Bucklew, J.A. (2004) Introduction in Rare Event Simulation. Springer-Verlag, New York.

[8] Dembo, A., Zeitouni, O. (1998) Large Deviations Techniques and Applications (2nd Edition). Springer-Verlag, New York.

[9] Iscoe, I., Ney, P., Nummelin, E. (1985). Large deviations of uniformly recurrent Markov additive processes. Adv. in Appl. Math. 6(4):373-412.

[10] Janssen, J., Manca, R. (2006) Applied Semi-Markov Processes. Springer, New York.

[11] Janssen, J., Manca, R. (2007) Semi-Markov Risk Models for Finance, Insurance and Reliability. Springer, New York.

[12] Kac, M. (1974) A stochastic model related to the telegrapher's equation. Rocky Mountain J. Math. 4:497-509.

[13] Lehtonen, T., Nyrhinen, H. (1992a). Simulating level crossing probabilities by importance sampling. Adv. in Appl. Probab. 24(4):858-874.

[14] Lehtonen, T., Nyrhinen, H. (1992b). On asymptotically efficient simulation of ruin probabilities in a Markovian environment. Scand. Actuarial J. 1992(1):60-75.

[15] Limnios, N., Oprişan, G. (2001) Semi-Markov Processes and Reliability. Birkhauser, Boston.

[16] Macci, C. (2009) Convergence of large deviation rates based on a link between wave governed random motions and ruin processes. Statist. Probab. Lett. 79(2):255-263.

[17] Mandjes, M. (2007) Large Deviations for Gaussian Queues. Modelling Communication Networks. John Wiley and Sons, Chichester.

[18] Mazza, C., Rullière, D. (2004) A link between wave governed random motions and ruin processes. Insurance Math. Econom. 35(2):205-222.

[19] Ney, P., Nummelin, E. (1987a) Markov additive processes I: eigenvalue properties and limit theorems. Ann. Probab. 15(2):561-592.

[20] Ney, P., Nummelin, E. (1987b) Markov additive processes II: large deviations. Ann. Probab. 15(2):593-607.

[21] Orsingher, E. (1990) Probability law, flow function, maximum distribution of wave governed random motions and their connections with Kirchoff's laws. Stochastic Process. Appl. $34(1): 49-66$.

[22] Rockafellar, R.T. (1970) Convex Analysis. Princeton University Press, New Jersey.

[23] Siegmund, D. (1976) Importance sampling in the Monte Carlo study of sequential test. Ann. Statist. 4(4):673-684.

[24] Stabile, G., Torrisi, G.L. (2010). Risk processes with non-stationary Hawkes claims arrivals. Methodol. Comput. Appl. Probab. 12(3):415-429.

[25] Willmot, G.E., Lin X.S. (2001) Lundberg Approximations for Compound Distributions with Insurance Applications. Lecture Notes in Statistics 156. Springer-Verlag, New York. 


\author{
Claudio Macci
}

\title{
Comments on the revised version
}

The author thanks the reviewers for their useful comments which led to an improvement of the presentation of the paper. Here it is the list of the main changes.

New structure of the paper (as a consequence of the comments of Referee 1). I presented the results (large deviation estimates for level crossing probabilities and importance sampling) in section 2 by referring to the general model close to the random walk proposed by Referee 1. The results were specialized to wave governed random motions driven by semi-Markov processes in section 3, where I also presented the simulations. Some parts of the Introduction of the previous version were postponed in section 3 . The final section 4 of this revised version coincides with section 5 of the previous version (in that section I did only some minor changes). I also inserted an Appendix at the end of the paper with an interesting large deviation principle.

Changes requested by Referee 1. I corrected the definition of $w$. I omitted the proof of Lemma 2.1 in the previous version, which is Lemma 3.1 in this revised version (I do not know if I understood well the comment on this point). I removed the symbols $\Psi_{N}(\ldots)$ and $\Upsilon_{A}(\ldots)$ used in the previous version for the level crossing probabilities.

Changes requested by Referee 2. I tried to take into account all the comments of Referee 2 . I rewrote the abstract by taking into account the changes in the presentation suggested by Referee 1. One could perform simulations by considering distribution functions $F_{1}$ and $F_{2}$ different from the ones concerning exponentially distributed random variables. However I prefer to avoid this because I guess that one can have closed formulas for $w(T)$ and $w$ only if $F_{1}$ and $F_{2}$ concern the case of exponentially distributed random variables.

Question of the Associate Editor. I presented a possible extension (of the framework of wave governed random motions driven by semi-Markov processes) in section 3 , where the state space of $J$ is $E=\{1, \ldots, s\}$ for some $s \geq 3$. 